\title{
ROLE OF THE AMINOTHIADIAZOLYL GROUP IN THE ANTIPSEUDOMONAL ACTIVITY OF CEFCLIDIN
}

\author{
NaO-AKI Watanabe and Isao Sugryama \\ Department of Microbiology and Infectious Diseases, \\ Tsukuba Research Laboratories, Eisai Co., Ltd., \\ 5-1-3 Tokodai, Tsukuba, Ibaraki 300-26, Japan
}

(Received for publication February 25, 1992)

\begin{abstract}
Cefclidin (E1040), which has an aminothiadiazolyl group in the $7 \beta$-side chain, showed about four-fold higher activity against Pseudomonas aeruginosa than its aminothiazolyl counterpart. Cefclidin had lower affinity and a higher $\mathrm{V}_{\max }$ value for the chromosomal type I $\beta$-lactamase (cephalosporinase) from $P$. aeruginosa than its aminothiazolyl counterpart. No differences between the affinities of both compounds for the most of the sensitive essential PBPs were observed. Hydrophilicity of cefclidin was higher than that of its counterpart. The antipseudomonal activity of cefclidin, which was increased by the introduction of the aminothiadiazolyl group, was suggested to have resulted mainly from higher resistance to cephalosporinase hydrolysis at pharmacologically relevant low concentrations due to its low affinity for cephalosporinase, and secondarily from good penetration of cefclidin through the outer membrane due to increased hydrophilicity.
\end{abstract}

Most cephalosporins possessing an $\alpha$-oxyimino-aminothiazolyl group in the $7 \beta$-side chain have broad antibacterial spectra being extended to Gram-negative bacteria, such as cefotaxime ${ }^{1)}$, ceftizoxime ${ }^{2)}$, and cefmenoxime $^{3}$. This heterocyclic group confers on cephalosporins a high affinity for penicillin-binding proteins (PBPs) and a high level of $\beta$-lactamase resistance, resulting in increased activity against Gram-negative bacteria except for Pseudomonas aeruginosa, as exhibited by cefotiam ${ }^{4)}$ and cefuroxime ${ }^{5}$. In addition, the presence of an aminothiazole side chain, as in ceftizoxime and cefotaxime, appears to improve the permeability of cefuroxime through the outer membrane ${ }^{6)}$.

Cefclidin (E1040), a new cephalosporin, showed higher activity against $P$. aeruginosa than ceftazidime ${ }^{7}$. This high activity against $P$. aeruginosa seemed to be associated with the introduction of an aminothiadiazolyl group in place of the aminothiazolyl group in the $7 \beta$-side chain of cefclidin ${ }^{8)}$. Recently,

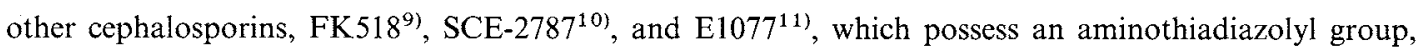
were also reported to show high activity against $P$. aeruginosa. However, the role of the aminothiadiazolyl group in the activity of cephalosporins against $P$. aeruginosa is unknown. In this study, we compared the antibacterial activity, the hydrolysis rate by cephalosporinase, and the affinity for penicillin-binding proteins of cefclidin with those of its aminothiazolyl counterpart (Fig. 1) in order to clarify the roles of this functional group.

Fig. 1. Chemical structures of cefclidin and its aminothiazolyl counterpart (compound 1).

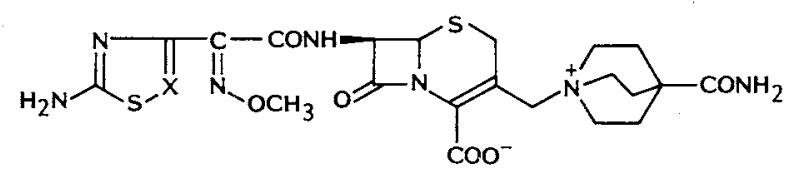

Cefclidin $\quad \mathrm{X}=\mathrm{N}$

Compound $1 \quad \mathrm{X}=\mathrm{CH}$ 


\section{Materials and Methods}

\section{Bacterial Strains}

Bacterial strains used in this study were recent clinical isolates of $P$. aeruginosa from various hospitals in Japan. The susceptibility breakpoint for ceftazidime was $\leq 6.25 \mu \mathrm{g} / \mathrm{ml}$. Strain E03441 SKR2 of $P$. aeruginosa was a mutant selected for ceftazidime resistance in vitro from a clinical isolate E03441 WT, and constitutively produced high levels of cephalosporinase ${ }^{12)}$. A laboratory strain of Escherichia coli was also used.

\section{Antibiotics and Media}

Cefclidin and its aminothiazolyl counterpart, compound 1, were synthesized at Tsukuba Research Laboratories of Eisai Co., Ltd. Ceftazidime and cephalothin were obtained commercially from Nippon Glaxo Co., Tokyo, Japan, and Shionogi Pharmaceutical Co., Osaka, Japan, respectively. Mueller-Hinton broth and agar (BBL Microbiology Systems, Cockeysville, Md, U.S.A.) were used for all experiments. MICs were determined by an agar dilution method. Approximately $5 \times 10^{4} \mathrm{cfu}$ per spot were inoculated onto agar plates that contained two-fold serial dilutions of antibiotics. The MIC was considered to be the lowest concentration of antibiotic that completely inhibited visible growth after incubation for 18 hours at $37^{\circ} \mathrm{C}^{7}$.

\section{Cephalosporinase Activity}

Cephalosporinase activity was measured with a Hitachi $220 \mathrm{~A}$ spectrophotometer by a spectrophotometric method ${ }^{13)}$. The reaction mixture consisted of $3 \mathrm{ml}$ of a substrate in $50 \mathrm{~mm}$ phosphate buffer $(\mathrm{pH} \mathrm{7.0)}$ and $50 \mu$ l of enzyme solution. Enzyme activity of crude cell extracts was determined with $100 \mu \mathrm{M}$ cephalothin as the substrate. One unit (U) represented $1 \mu \mathrm{mol}$ of cephalothin hydrolyzed per minute. The enzyme reaction was carried out at $30^{\circ} \mathrm{C}$. The $\mathrm{Km}$ and $\mathrm{Ki}$ values were calculated from Lineweaver-Burk and Dixon plots, respectively. For the determination of the $K m$ and $K i$ values, partially purified enzyme was used. Cephalosporinase from $P$. aeguginosa E03441 SKR2 was prepared by sonication, CM-Sephadex C-50 ion-exchange chromatography, and Sephadex G-100 gel filtration as described previously ${ }^{14)}$. The spectral parameters used in this study were as follows: Cefclidin, $264.5 \mathrm{~nm}, \Delta \varepsilon=8.64 \mathrm{~mm}^{-1} \mathrm{~cm}^{-1}$; compound 1, $264 \mathrm{~nm}, \Delta \varepsilon=7.43 \mathrm{mM}^{-1} \mathrm{~cm}^{-1}$; ceftazidime, $260 \mathrm{~nm}, \Delta \varepsilon=9.59 \mathrm{~mm}^{-1} \mathrm{~cm}^{-1}$; cephalothin, $262 \mathrm{~nm}$, $\Delta \varepsilon=7.66 \mathrm{mM}^{-1} \mathrm{~cm}^{-1}$. The protein concentration was determined by the method of LowRY et al. ${ }^{15)}$ with bovine serum albumin as the standard.

\section{Cephalosporinase Induction}

An overnight culture of the test strain was diluted 20 -fold into fresh Mueller-Hinton broth and incubated with shaking at $37^{\circ} \mathrm{C}$. After 3 hours of incubation, inducers, cefclidin, compound 1 , or ceftazidime, were added. Incubation was continued for 2 hours, and the cells were harvested and washed twice with $50 \mathrm{~mm}$ phosphate buffer ( $\mathrm{pH} \mathrm{7.0)}$. The cells were suspended in the same buffer and disrupted with a model 350 Sonifier (Branson Sonic Power Co., Banbury, Conn., U.S.A.) in an ice-water bath. Cell debris and unbroken cells were removed by centrifugation, and the resulting supernatant fluid was used as crude enzyme. Cephalosporinase activity of the crude extracts was determined as described above.

Isoelectric focusing of the supernatants from centrifuged cell sonic extracts was performed on an Ampholine PAG plate (Pharmacia; $\mathrm{pH}$ range, $3.5 \sim 9.5)$ at $10^{\circ} \mathrm{C}$. Samples $(10 \mu \mathrm{g}$ in $2 \mu \mathrm{l})$ were loaded and run at $30 \mathrm{Watt}$ for 1.5 hours. $\beta$-Lactamase activity was visualized by overlaying the gel with $1.5 \%$ agar containing $25 \mathrm{~mm}$ phosphate buffer (pH 7.0) and $50 \mu \mathrm{g}$ of nitrocefin per $\mathrm{ml}$.

\section{Affinity for PBPs}

The affinities of cefclidin and compound 1 for PBPs were determined by the conventional competition assay with $\left[{ }^{14} \mathrm{C}\right]$ benzylpenicillin (Amersham Japan, Tokyo, Japan). The competition assay was carried out as described by NoGUCHI et al. ${ }^{16)}$. Membranes were prepared by differential centrifugation of the sonic extracts, resuspended in $50 \mathrm{mM}$ phosphate buffer $(\mathrm{pH} 7.0)$ containing $10 \mathrm{mM} \mathrm{MgCl}_{2}$, and stored at $-80^{\circ} \mathrm{C}$ until used in PBP binding experiments. Membrane proteins $(300 \mu \mathrm{g}$ in $30 \mu \mathrm{l})$ were incubated with various concentrations of antibiotics $(3 \mu \mathrm{l})$ for 10 minutes at $30^{\circ} \mathrm{C}$. $\left[{ }^{14} \mathrm{C}\right]$ benzylpenicillin $(0.15 \mu \mathrm{Ci}$ in $3 \mu \mathrm{l})$ was added, 
and incubation was continued for an additional 10 minutes. Excess unlabeled benzylpenicillin was added to stop the reaction, and the membranes were solubilized in $1 \%$ sodium lauroyl sarcosinate. Sarcosinate-soluble fractions were subjected to $0.1 \%$ sodium dodecyl sulfate-10\% polyacrylamide gel electrophoresis. For fluorograpy, gels were incubated for 1 hour in $\mathrm{En}^{3} \mathrm{Hance}$ (Daiichi Pure Chemicals Co., Tokyo, Japan), soaked in water, dried, and exposed to X-ray film at $-80^{\circ} \mathrm{C}$ for about 1 month. PBP fluorography was quantitated with a model CS-9000 flying-spot scanner (Shimadzu, Kyoto, Japan) to generate areas under the peaks corresponding to the various PBPs at each antibiotic concentration. The binding affinities of antibiotics for each PBP were expressed in terms of the concentration required to reduce $\left[{ }^{14} \mathrm{C}\right]$ benzylpenicillin binding by $50 \%$.

\section{High Performance Liquid Chromatography (HPLC)}

The hydrophilicities of $\beta$-lactams were compared as the retention time on HPLC. HPLC was performed at a flow rate of $1 \mathrm{ml} /$ minute with a system LC-6A (Shimadzu) apparatus. Chromatography analyses were carried out on a reverse-phase Inertsil ODS-2 analytical column $(4.6 \times 150 \mathrm{~mm}$; GL Sciences Co., Tokyo, Japan). The mobile phase consisted of $\mathrm{H}_{2} \mathrm{O}, \mathrm{CH}_{3} \mathrm{OH}$, and $\mathrm{CH}_{3} \mathrm{COONH}_{4}(900(\mathrm{v}): 100(\mathrm{v}): 1(\mathrm{w}))$. $\beta$-Lactams were detected at $254 \mathrm{~nm}$ with a model SPD-6A UV detector (Shimadzu).

\section{Results}

Antipseudomonal Activity of Cefclidin and Compound 1

The $\mathrm{MIC}_{50}$ and $\mathrm{MIC}_{90}$ values of cefclidin for ceftazidime-susceptible strains of $P$. aeruginosa were 0.78 and $1.56 \mu \mathrm{g} / \mathrm{ml}$, respectively. This activity was four times higher than those of its aminothiazolyl counterpart (compound 1) and ceftazidime. Although the activity of cefclidin against ceftazidime-resistant strains was four-fold lower than against ceftazidime-susceptible strains, cefclidin showed 4-fold and 16 -fold

Table 1. In vitro activity of cefclidin and compound $\mathbf{1}$ against clinical isolates of $P$. aeruginosa

\begin{tabular}{clccc}
\hline \multirow{2}{*}{ Strain (No.) } & & \multicolumn{3}{c}{ MIC $(\mu \mathrm{g} / \mathrm{ml})$} \\
\cline { 3 - 4 } & & Range & $50 \%$ & $90 \%$ \\
\hline Ceftazidime-susceptible (27) & Cefclidin & $0.20 \sim 3.13$ & 0.78 & 1.56 \\
& Compound 1 & $0.78 \sim 12.5$ & 3.13 & 6.25 \\
& Ceftazidime & $0.78 \sim 6.25$ & 3.13 & 6.25 \\
Ceftazidime-resistant (25) & Cefclidin & $0.78 \sim 12.5$ & 3.13 & 12.5 \\
& Compound 1 & $6.25 \sim 50$ & 12.5 & 50 \\
& Ceftazidime & $25 \sim>100$ & 50 & 100 \\
\hline
\end{tabular}

Table 2. MICs and $\beta$-lactamase levels in $E$. coli and $P$. aeruginosa strains.

\begin{tabular}{|c|c|c|c|c|c|}
\hline \multirow{2}{*}{ Strain } & \multirow{2}{*}{ Inducer $^{\mathrm{a}}$} & \multicolumn{3}{|c|}{$\operatorname{MIC}(\mu \mathrm{g} / \mathrm{ml})$} & \multirow{2}{*}{$\begin{array}{l}\beta \text {-Lactamase specific activity } \\
(\mu \mathrm{mol} / \text { minute/mg-protein })^{\mathrm{b}}\end{array}$} \\
\hline & & Cefclidin & Compound $\mathbf{1}$ & Ceftazidime & \\
\hline \multicolumn{6}{|l|}{ E. coli } \\
\hline NIHJ JC-2 & - & 0.05 & 0.05 & 0.20 & ND \\
\hline \multicolumn{6}{|l|}{$P$. aeruginosa } \\
\hline E03441 WT & - & 0.78 & 1.56 & 3.13 & 0.0037 \\
\hline E03441 SKR2 & - & 3.13 & 12.5 & 50 & 0.748 \\
\hline E03441 WT & + & 3.13 & 12.5 & 50 & 0.675 \\
\hline E03441 SKR2 & + & 6.25 & 25 & 100 & 1.33 \\
\hline
\end{tabular}

a Cells grown for 2 hours in $0.10 \mu \mathrm{g}$ of imipenem per $\mathrm{ml}$.

b Measured in supernatant of sonic extract with $100 \mu \mathrm{M}$ cephalothin as the substrate.

ND: Not detected $(<0.001 \mu \mathrm{mol} / \mathrm{minute} / \mathrm{mg})$. 
higher activity than did compound $\mathbf{1}$ and ceftazidime, respectively (Table 1). Ceftazidime-resistant strains produced cephalosporinase at about 0.1 to $8 \mathrm{U} / \mathrm{mg}$ of protein without inducer agents, whereas ceftazidime-susceptible strains produced very low levels of cephalosporinase (less than $0.01 \mathrm{v} / \mathrm{mg}$ of protein).

Of these strains, strain E03441 WT was chosen as the representative ceftazidime-susceptible strain. The susceptibility of E03441 WT and its cephalosporinase-derepressed mutant E03441 SKR2 to $\beta$-lactam antibiotics and levels of cephalosporinase produced by each strain are shown in Table 2.

No differences between the outer membrane profiles of both strains were observed (data not shown). Strain E03441 WT produced a very low level of cephalosporinase scarcely detectable in the absence of inducer $(0.10 \mu \mathrm{g} / \mathrm{ml}$ imipenem). Imipenem was a potent inducer, increasing the enzyme activity about 200 times. This level was comparable to that of the derepressed mutant E03441 SKR2 in the uninduced state. The strain E03441 SKR2 had only a two-fold increase in enzyme activity when induced by imipenem, suggesting that the high levels of cephalosporinase in E03441 SKR2 were constitutively produced. The higher cephalosporinase levels were accompanied by 16 - to 32 -fold increases in the ceftazidime MICs and by 8 - to 16-fold increases in the compound 1 MICs. Cefclidin MICs increased four-fold for strains with higher cephalosporinase levels. Against $E$. coli NIHJ JC-2, the activity of cefclidin was comparable to that of compound 1.

\section{Interaction of Cefclidin and Compound 1 with Cephalosporinase}

Cefclidin, compound $\mathbf{1}$, and ceftazidime showed little enhancement of the enzyme activity of E03441 WT at $1 \mu \mathrm{g} / \mathrm{ml}$, whereas induction by compound 1 at $\geq 10 \mu \mathrm{g} / \mathrm{ml}$ was as high as that by cefclidin and ceftazidime. Thus, no significant differences in the ability to induce enzyme activity was observed between cefclidin, compound 1, and ceftazidime (Table 3). Isoelectric focusing of the supernatants from cell sonic extracts of strains E03441 WT and E03441 SKR2 in the induced or uninduced state yielded a main nitrocefin-positive band with a pI of 8.8 .

Hydrolysis rates of cefclidin, compound $\mathbf{1}$, and ceftazidime by cephalosporinase from strain E03441 SKR2 decreased significantly as substrate concentrations decreased. Cefclidin and compound 1 were hydrolyzed more rapidly than ceftazidime at $100 \mu \mathrm{M}$, which is the concentration commonly used in testing susceptibility to $\beta$-lactamase. The $\mathrm{V}_{\max }$ value of cefclidin was about two times that of compound 1 ,

Table 3. Induction of $\beta$-lactamase in strain E03441 WT by $\beta$-lactams.

\begin{tabular}{lcccc}
\hline & \multicolumn{3}{c}{$\begin{array}{c}\beta \text {-Lactamase specific activity } \\
\text { (nmol/minute/mg-protein) } \\
\text { at the following inducer } \\
\text { concentrations }(\mu \mathrm{g} / \mathrm{ml})\end{array}$} \\
\cline { 2 - 5 } & 0 & 1 & 10 & 100 \\
\hline None & 3.73 & & & \\
Cefclidin & & 4.39 & 281 & 646 \\
Compound 1 & & 5.55 & 383 & 466 \\
Ceftazidime & & 6.31 & 153 & 424 \\
\hline
\end{tabular}

Table 4. Hydrolysis of $\beta$-lactams by cephalosporinase from strain E03441 SKR2.

\begin{tabular}{lccccc}
\hline Compound & $\begin{array}{c}\mathrm{V}_{\max } \\
\text { (nmol/minute/mg- } \\
\text { protein) }\end{array}$ & $\begin{array}{c}K m \\
(\mu \mathrm{M})\end{array}$ & $\begin{array}{c}K i \\
(\mu \mathrm{M})\end{array}$ & \multicolumn{2}{c}{$\begin{array}{c}\text { Hydrolysis rate (nmol/minute/mg-protein) } \\
\text { at } 2 \mu \mathrm{M}^{\mathrm{a}}\end{array}$} \\
\hline Cefclidin & 0.467 & 941 & $>1,000$ & 0.00099 & 0.0419 \\
Compound 1 & 0.251 & 184 & 108 & 0.0027 & 0.0873 \\
Ceftazidime & 0.0216 & 8.31 & 6.85 & 0.0042 & 0.0193 \\
Cephalothin & 1038 & 68.5 & - & 29.5 & 582 \\
\hline
\end{tabular}

a Calculated from Michaelis-Menten equation ${ }^{13)}$ for a substrate concentration of $2 \mu \mathrm{M}$. 
Table 5. Affinity of cefclidin and compound 1 for PBPs of strain E03441 WT.

\begin{tabular}{lcccccccc}
\hline \multirow{2}{*}{ Compound } & \multicolumn{4}{c}{$\mathrm{I}_{50}(\mu \mathrm{g} / \mathrm{ml})^{\mathrm{a}}$} & & MIC \\
\cline { 2 - 6 } & PBP 1A & PBP 1B & PBP 2 & PBP 3 & PBP 4 & PBP 5 & $(\mu \mathrm{g} / \mathrm{ml})$ \\
\hline Cefclidin & 3.13 & 50 & $>50$ & 0.20 & 12.5 & $>50$ & 0.78 \\
Compound 1 & 0.78 & 50 & $>50$ & 0.20 & 3.13 & $>50$ & 1.56 \\
\hline
\end{tabular}

a $\mathrm{I}_{50}$ : Concentration required to prevent $\left[{ }^{14} \mathrm{C}\right]$ benzylpenicillin binding by $50 \%$.

and was 22 times that of ceftazidime. Furthermore, The $K m$ value of cefclidin was about 5 times and 110 times greater than those of compound 1 and ceftazidime, respectively. The $K i$ value of cefclidin was nearly one order of magnitude and at least two orders of magnitude greater than those of compound 1 and ceftazidime, respectively (Table 4).

For comparison of the hydrolysis rates of these three cephalosporins at the cellular level, that is at a concentration near the MIC, the rates for $2 \mu \mathrm{M}$ substrate (corresponding to about $1 \mu \mathrm{g} / \mathrm{ml}$ ) were estimated from the Michaelis-Menten equation ${ }^{17)}$ using $\mathrm{V}_{\max }$ and $K m$ values. At $2 \mu \mathrm{M}$, the hydrolysis rate of cefclidin, which was about three- to four-fold lower than those of compound 1 and ceftazidime, was about $0.2 \%$ of $\mathrm{V}_{\max }$, whereas the rates of compound 1 and ceftazidime were about $1 \%$ and $19 \%$ of the corresponding $\mathrm{V}_{\max } \mathrm{s}$, respectively.
Fig. 2. Fluorographs of $P$. aeruginosa E03441 WT isolated membranes in PBP competition experiments with cefclidin (A) and compound 1 (B).
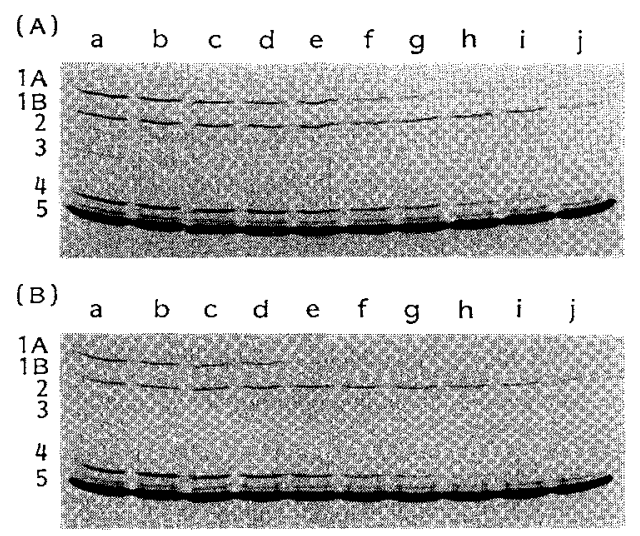

PBP designations are indicated on the left side of each panel. The concentrations of the competing antibiotics in lanes a to $\mathrm{j}$ are $0,0.20,0.39,0.78,1.56$, $3.13,6.25,12.5,25$, and $50 \mu \mathrm{g} / \mathrm{ml}$, respectively.

\section{Affinity for PBPs}

Affinities of cefclidin and compound 1 for PBPs of strain E03441 WT were estimated by measuring the competition of both compounds with $\left[{ }^{14} \mathrm{C}\right]$ benzylpenicillin for binding to PBPs. The results of these competition experiments are shown in Fig. 2. The results indicate that, qualitatively, compound 1 binds to PBP 3 as well as cefclidin, and that binding to PBP 3 is evident.

Densitometry was used to quantify the binding affinities of these two cephalosporins for the individual PBPs. Both cephalosporins exhibited poor binding to PBPs $1 \mathrm{~B}, 2$, and $5\left(\mathrm{I}_{50} \geq 50 \mu \mathrm{g} / \mathrm{ml}\right)$, and high binding to PBP 3, for which $\mathrm{I}_{50}$ s of cefclidin and compound 1 were virtually identical. However, for PBPs $1 \mathrm{~A}$ and 4 , compound 1 showed a four-fold lower $I_{50}$ value than cefclidin.

\section{Hydrophilicity of Cefclidin and Compound 1}

Hydrophilicity of cephalosporins was qualitatively estimated by measuring their retention times on a reverse-phase HPLC column. Retention times of cefclidin, compound 1, and ceftazidime were 3.8, 5.0, and 5.9 minutes, respectively. This result indicates that the hydrophilicity of cefclidin is higher than those of compound 1 and ceftazidime. 


\section{Discussion}

Cefclidin exhibited about four-fold higher activity against $P$. aeruginosa than its aminothiazolyl counterpart (compound 1). With respect to the antipseudomonal activity of cefclidin, the effects of the aminothiadiazolyl group are summarized as follows: (i) Decreased affinity for the chromosomal cephalosporinase, but increased maximal hydrolysis rate by cephalosporinase; (ii) high affinity for PBP 3 in spite of decreased affinity for PBPs 1A and 4; (iii) increased hydrophilicity.

In general, three factors determine the efficacy of $\beta$-lactams against Gram-negative bacteria; outer membrane permeability, resistance to periplasmic $\beta$-lactamase, and affinity for the target PBPs. $P$. aeruginosa is intrinsically resistant to most $\beta$-lactams, and this has been considered to be largely due to the poor permeability of the outer membrane. The outer membrane permeability of $P$. aeruginosa for $\beta$-lactams is at least two orders of magnitude lower than that of $E$. coli ${ }^{6}$. Firstly, therefore, it is assumed that the improved antipseudomonal activity of cefclidin may be the result of permeability through the outer membrane, owing to its increased hydrophilicity. Although the pathway of cephalosporins in $P$. aeruginosa is still disputed ${ }^{18,19)}$, the dipolar ionic $\beta$-lactams such as cefclidin and compound 1 penetrate through porin channels in $E$. coli much more rapidly than $\beta$-lactams with a net negative charge such as ceftazidime ${ }^{20)}$. However, the permeation of the former group is much less affected by the hydrophobicity of the solutes than that of the latter group, and the permeability of porin channels in $P$. aeruginosa is much less affected by hydrophobicity than that in $E$. coli ${ }^{6}$. If so, significant differences between the penetration rate of cefclidin and that of compound 1 in $P$. aeruginosa are not likely, since both cephalosporins possess the same electrical charge and almost the same molecular weight (550.6 and 549.6, respectively).

The second important factor in antipseudomonal activity is the interaction of $\beta$-lactams with $\beta$-lactamase. $P$. aeruginosa produces chromosomal inducible or derepressed cephalosporinase. By the introduction of the aminothiadiazolyl group, the $V_{\max }$ value of cefclidin was increased to about two times that of compound $\mathbf{1}$, and the $\mathrm{Km}$ value of cefclidin was increased to about three times that of compound $\mathbf{1}$, indicating that cefclidin was hydrolyzed about three times more slowly than was its aminothiazolyl counterpart by $P$. aeruginosa enzyme when the hydrolysis rates at a substrate concentration of $2 \mu \mathrm{M}$ were calculated on the basis of kinetic data. Although it was presumed that the affinity of cefclidin for PBPs may be reduced since PBPs possess similar active sites to $\beta$-lactamases, cefclidin had the same affinity level for PBP 3, one of the essential PBPs, as compound 1, but the affinity of cefclidin for PBPs 1A and 4 was reduced. Therefore, cefclidin had as high an affinity for the most sensitive of the essential PBPs as compound 1.

In conclusion, the aminothiadiazolyl group in the $7 \beta$-side chain appears to confer antipseudomonal activity on cefclidin, resulting mainly from the reduced affinity for the chromosomal cephalosporinase coupled with high resistance to enzymatic hydrolysis. Moreover, the effect of the aminothiadiazolyl group is more evident against the cephalosporinase-overproducing strain than against the inducible strain. In contrast, against $E$. coli which constitutively produces only a very low level of cephalosporinase and has higher permeability of the outer membrane for newer $\beta$-lactams than $P$. aeruginosa, the aminothiadiazolyl group had little effect on activity. In addition, good penetrability of cefclidin may participate in part in its good antipseudomonal activity since the hydrophilicity of cefclidin is higher than those of compound 1 and ceftazidime.

\section{References}

1) Neu, H. C.; N. AswapokeE, P. Aswapokee \& K. P. FU: HR 756, a new cephalosporin active against gram-positive and gram-negative aerobic and anaerobic bacteria. Antimicrob. Agents Chemother. 15: 273 281, 1979

2) Kamimura, T.; Y. Matsumoto, N. Okada, Y. Mine, M. Nishida, S. Goto \& S. Kuwahara: Ceftizoxime (FK 749), a new cephalosporin: in vitro and in vivo antibacterial activities. Antimicrob. Agents Chemother. 16:540 548, 1979

3) TsuchiYa, K.; M. Kondo, M. Kida, M. NAKaO, T. Iwahi, T. Nish, Y. NoI, M. Takeuchi \& Y. Nozaki: Cefmonoxime (SCE-1365), a novel broad-spectrum cephalosporin: in vitro and in vivo antibacterial activities. Antimicrob. Agents Chemother. 19: 56 65, 1981

4) NOZAKI, Y.; A. IMADA \& M. YONEDA: SCE-963, a new potent cephalosporin with high affinity for penicillin-binding proteins 1 and 3 of Escherichia coli. Antimicrob. Agents Chemother. 15: 20 27, 1979

5) O'Callaghan, C. H.; R. B. Sykes, A. Griffiths \& J. E. Thornton: Cefuroxime, a new cephalosporin antibiotic: 
activity in vitro. Antimicrob. Agents Chemother. 9: $511 \sim 519,1976$

6) Nikaido, H.: Role of the outer membrane of gram-negative bacteria in antimicrobial resistance. Handb. Exper. Pharmacol. 91: $119 \sim 245,1983$

7) Watanabe, N.; K. Katsu, M. Moriyama \& K. KIroh: In vitro evaluation of E1040, a new cephalosporin with potent antipseudomonal activity. Antimicrob. Agents Chemother. 32: 693 701, 1988

8) Sugtyama, I.; Y. Komatsu \& H. Yamauchi: Synthesis and structure-activity relationships of a new series of cephalosporins, E1040 and related compounds. J. Antibiotics 45: 103 112, 1992

9) Sakane, K.; K. Kawabata, Y. Inamoto, S. Okuda, T. Kamimura \& T. Takaya: FK518, a new parenteral antipseudomonal cephalosporin I. Synthesis and structure-activity relationships of 3-aminopyrazoliomethyl cephalosporins. Program and Abstracts of the 30th Intersci. Conf. on Antimicrob. Agents Chemother., No. 454, p. 162, Atlanta, Oct. $21 \sim 24,1990$

10) Miyake, A.; Y. Yoshimura, M. Yamaoka, T. Nishimura, N. Hashimoto \& A. Imada: Synthesis and structure-activity relationships of 3-condensed azoliummethyl cephalosporins including SCE-2787, a new parenteral cephalosporin. Program and Abstracts of the 29th Intersci. Conf. on Antimicrob. Agents Chemother., No. 486, p. 182, Houston, Sept. $17 \sim 20,1989$

11) KamiYa, T.; T. Naito, Y. Kai, Y. Komatsu, M. Sasho, N. Sato, T. Nakamura, S. Negi, Y. Machida \& H. YAMAUCHI: Synthesis and structure-activity relationships of fluoromethoxyimino containing cephems; E1077, a novel parenteral cephem. Program and Abstracts of the 30th Intersci. Conf. on Antimicrob. Agents Chemother., No. 447 , p. 160 , Atlanta, Oct. $21 \sim 24,1990$

12) Watanabe, N.\& K. Katsu: Bactericidal activity of cefclidin (E1040), a new cephalosporin, against Pseudomonas aeruginosa under conditions simulating plasma pharmacokinetic: no development of chromosomal resistance to $\beta$-lactams. J. Antimicrob. Chemother, in press

13) SAMUNI, A.: A direct spectrophotometric assay and determination of Michaelis constants for $\beta$-lactamase reaction. Anal. Biochem. 63: 17 26, 1975

14) Murata, T.; S. Minami, K. Yasuda, S. Iyobe, M. Inoue \& S. Mitsuhashi: Purification and properties of cephalosporinase from Pseudomonas aeruginosa. J. Antibiotics 34: 1164 1170, 1981

15) Lowry, O. H.; N. J. Rosebrough, A. L. Farr \& R. J. Randall: Protein measurement with the Folin phenol reagent. J. Biol. Chem. 193: 265 275, 1951

16) Noguchi, H.; M. Matsuhashi \& S. Mrtsuhashi: Comparative studies of penicillin-binding proteins in Pseudomonas aeruginosa and Escherichia coli. Eur. J. Biochem. 100: 41 49, 1979

17) Vu, H. \& H. Nikaido: Role of $\beta$-lactam hydrolysis in the mechanism of resistance of a $\beta$-lactamase-constitutive Enterobacter cloacae strain to expanded-spectrum $\beta$-lactams. Antimicrob. Agents Chemother. 27: 393 398, 1985

18) SATAKE, S.; E. YoshiHara \& T. NAKAE: Diffusion of $\beta$-lactam antibiotics through liposome membranes reconstituted from purified porins of the outer membrane of Pseudomonas aeruginosa. Antimicrob. Agents Chemother. 34: $685 \sim 690,1990$

19) Nikaido, H.; K. Nikaido \& S. Harayama: Identification and characterization of porins in Pseudomonas aeruginosa. J. Biol. Chem. 266: 770 779, 1991

20) Yoshimura, F, \& H. Nikaido: Diffusion of $\beta$-lactam antibiotics through the porin channels of Escherichia coli K-12. Antimicrob. Agents Chemother. 27: 84 92, 1985 